\title{
COVID-19 Lethality in Sub-Saharan Africa and Helminth Immune Modulation
}

\author{
Luis Fonte $^{1 *}$, Armando Acosta ${ }^{2 *}$, Maria E. Sarmiento ${ }^{2}$, María Ginori ${ }^{3}$, Gissel García ${ }^{4}$ and \\ Mohd Nor Norazmi ${ }^{2 *}$ \\ ${ }^{1}$ Department of Parasitology, Institute of Tropical Medicine "Pedro Kouri", Havana, Cuba, ${ }^{2}$ School of Health Sciences, \\ Universiti Sains Malaysia, Kelantan, Malaysia, ${ }^{3}$ Department of Teaching, Polyclinic "Plaza de la Revolución", Havana, Cuba, \\ ${ }^{4}$ Department of Medical Genetic, Hospital "Hermanos Ameijeiras", Havana, Cuba
}

Keywords: Sub-Saharan Africa, helminth immune modulation, SARS CoV-2, COVID-19, lethality

OPEN ACCESS

Edited by:

Paul Giacomin,

James Cook University, Australia

Reviewed by:

Keke Celeste Fairfax,

The University of Utah, United States

William Horsnell,

University of Cape Town, South Africa Jürgen Schwarze,

The University of Edinburgh,

United Kingdom

${ }^{*}$ Correspondence:

Luis Fonte

luisfonte@infomed.s/d.cu

Armando Acosta

armando@usm.my

Mohd Nor Norazmi

norazmimn@usm.my

Specialty section:

This article was submitted to

Microbial Immunology,

a section of the journal

Frontiers in Immunology

Received: 22 June 2020

Accepted: 04 September 2020

Published: 08 October 2020

Citation:

Fonte L, Acosta A, Sarmiento ME, Ginori M, García G and Norazmi MN (2020) COVID-19 Lethality in

Sub-Saharan Africa and Helminth Immune Modulation.

Front. Immunol. 11:574910.

doi: 10.3389/fimmu.2020.574910
The acronym COVID-19 (Coronavirus Disease 2019) identifies the human disease caused by the Severe Acute Respiratory Syndrome Coronavirus-2 (SARS-CoV-2) (1). Since the outbreak of COVID-19 in Wuhan city, China, in December 2019, it has rapidly spread through 185 countries in all continents (2). SARS CoV-2 infection, and its related disease, is now a major health problem, with 23,057,288 infected individuals and 800,906 deaths confirmed worldwide as of August 24, 2020 (3).

The natural progression of SARS CoV-2 infection is extremely variable. It ranges between an asymptomatic course or mild clinical expression, which generally occurs in children and healthy adults, and the development of pneumonia and severe multi-organ failure, more frequent in the elderly and in patients of chronic diseases. This broad spectrum of clinical expression is the consequence of another one at immunological level: SARS CoV-2 infection activates innate and adaptive immune responses that, in the most frequent and benign of evolutions, lead to the containment of viral replication and recovery and, in the most unfavorable of sequences, can stimulate an intense pulmonary inflammatory reaction that, leading to more severe complications, can end in death (4).

SARS-CoV-2, unlike its close genetic relative SARS CoV, has the ability to infect and reproduce in the upper respiratory tract (5). There, type I/III interferons, tumor necrosis factor alpha (TNF- $\alpha-$ ) interleukin-1 (IL-1), IL-6, and IL-18, among other components of the innate immunity, control the infection in the majority of the individuals (6). However, if SARS-CoV-2 passes through that first control and spreads, along the conducting airways, to the alveoli, it can replicate there more rapidly, causing pneumonia and other severe clinical complications (7).

Severe COVID-19 evolution is associated with an increase in the proportion of Th1 and Th17 cells, with their corresponding cytokines IFN- $\gamma$, IL17, IL-23, and TNF- $\alpha$ (8). At the same time, there is an activation of the inflammatory CD14+CD16+ monocytes, with an amplified production of cytokines, such as IL-6, and chemokines, such as CC- chemokine ligand 2 (CCL2), CCL3 and CXC- chemokine ligand 10 (CXCL10) $(8,9)$. The triggering of these cellular types, and the release of their mediators, leads to an increase of inflammation, vascular permeability and leakage with severe lung damage (8).

COVID-19 has shown significant differences in its lethality rate between continents, regions and countries (3). Of them, the more notable is the higher rates registered in economically developed regions with robust health systems, such as Europe and the United States, compared to countries having poor economies and insufficient health services, in particular, almost all the nations that constitute the Sub-Saharan Africa (SSA) (Table 1) (3). Some factors, or combinations of them, have been mentioned to explain the unexpected evolution: diagnostic test unavailability, age and genetic background of the population, mutational variations of SARS-CoV-2 in relation with 
TABLE 1 | COVID-19 lethality rates in Europe, United States and Sub-Saharan Africa as of August 23, 2020.

\begin{tabular}{lccc}
\hline Region & Confirmed cases & Deaths & Lethality (\%) \\
\hline Europe & $3,970,890$ & 216,478 & 5.45 \\
United States & $5,567,217$ & 174,246 & 3.12 \\
Sub-Saharan Africa & 959,311 & 18,897 & 1.96 \\
\hline
\end{tabular}

Source: (3).

Confirmed Case: person positive by Polymerase Chain Reaction (PCR) test for SARS CoV-2.

COVID-19 death: a COVID-19 death is defined as a death resulting from a clinically compatible illness in a probable or confirmed COVID-19 case, unless there is a clear alternative cause of death that cannot be related to COVID-19 disease (e.g., trauma).

There should be no period of complete recovery between the illness and death.

geographic settings, environmental temperature and humidity non-favorable for viral replication, BCG vaccination policies and endemicity of other infections (10-12). Here, we hypothesize the possible role of helminth immune modulation in the low COVID-19 lethality in SSA.

In 2009, Hotez and Kamath, in a landmark paper analyzed the striking connection between living conditions and prevalence of Neglected Tropical Diseases (NTDs), linking the world's greatest concentration of poverty with helminth infection prevalence in SSA region (13). In this region, " $73 \%$ of the population lives on <US\$2 per day, the most common NTDs, such as the soil-transmitted helminth infections, schistosomiasis, lymphatic filariasis and onchocerciasis, affected more than 500 million people" (13). For example, "of the world's 207 million estimated cases of schistosomiasis, 93\% occur in SSA (192 million)" (13). Since then, little has changed in that part of the planet.

The "equilibrium" occurring in individuals chronically infected with helminths is the result of hundreds of millions of years of host-parasite coevolution. That prolonged interaction has led to the development of defensive responses by the human hosts and to the achievement of complex immune modulatory means by the helminths. The host protective responses against helminths, which are multicellular and large organisms, include wound repair mechanisms, which reduce the tissue damage that these parasites may cause as they move through body organs.

The cellular damage resulting from helminths migration through tissues is the major stimulus of the innate immunity against those parasites, as danger associated molecular patterns (DAMPs) are released and induce the production of cytokine alarmins (IL-25, IL-33, and thymic stromal lymphopoietin -TSLP-) by epithelial cells (14). IL-25 and IL-33 trigger the production of IL-4, IL-5 and IL-13, the principal mediators of type 2 responses, by type 2 innate auxiliary cells (14). On the other hand, TSLP limits IL-12 production by dendritic cells, the main promoter of type 1 responses (15).

For controlling the helminth infections, the adaptive immunity of the host usually develops type 2 immune responses, including the development of Th2 cells and the release of cytokines such as IL-4, IL-5, and IL-13 (16). This host-helminth interaction has, at least, two additional outcomes: (i) the classical and best-known down-regulation of type Th1 and type Th17 responses (and its related cytokines IL-12, IFN- $\gamma$, IL17, IL-23, TNF- $\alpha$ ) by the Th2 cytokines $(16,17)$ and (ii) the helminths limitation of both host type 1 and type 2 responses by enhancing FOXP3 + T regulatory cells, B regulatory cells and alternatively activated macrophages (AAMs) activities, which together cause the release of regulatory cytokines such as IL-10 and transforming growth factor (TGF- $\beta$ ) (18).

The modulation by helminths of the immune responses of their hosts has relevant clinical and epidemiological consequences: increased susceptibility to some infections, decreased frequency and intensity of allergic, autoimmune and inflammatory diseases, inadequate responses to vaccines and, as is possible in the case of SARS-CoV-2 infection, may inhibit the inflammatory processes that characterize infection by other microorganisms (17).

Helminths modulation has the ability to suppress inflammatory responses present during infection by protozoon, bacteria and virus: (i) when Plasmodium falciparum infection occurs in an individual infected with helminths, the effects of pro-inflammatory cytokines (IFN- $\gamma$ and TNF- $\alpha$ ) that characterize severe forms of malaria are attenuated by the action of anti-inflammatory mediators (IL-10 and TGF- $\beta$ ) and, consequently, decrease the chances of developing severe inflammatory conditions, including cerebral malaria (19); (ii) mice infected by Nippostrongylus brasiliensis showed increased susceptibility to Mycobacterium tuberculosis. Apparently, AAMs with impaired killing capacity in a less inflammatory Type 2 pulmonary milieu function as a mycobacteria reservoir (20); (iii) Trichinella spiralis infection limits inflammatory pulmonary damage induced by influenza virus in mice (21).

Nevertheless, and analyzing the helminth-virus relationship from a more holistic perspective, it is necessary to mention that helminths can enhance anti-viral mechanisms leading to a better control of viral load. Two examples: (i) during helminth infection IL-4 can expand and condition virtual memory CD8+ $\mathrm{T}$ cells ( $\mathrm{T}_{\mathrm{VM}}$ cells) for more rapid $\mathrm{CD} 8$ responses against subsequent cognate antigen encounter. Apparently, immunity against helminths has evolved a safety mechanism through induction of highly responding $\mathrm{T}_{\mathrm{VM}}$ cells to counterbalance anti-inflammatory effects related to type 2 immunity on the development of effective antiviral responses (22); (ii) mice infection by the rodent roundworm, Heligmosomoides polygyrus, significantly reduce pulmonary lung damage and viral load following intranasal infection with respiratory syncytial virus. Interestingly, those effects were independent of adaptive immune responses because protection was lost in germ free mice, denoting a possible role of intestinal microbiota (23).

Taking into account the arguments described above, it is plausible to consider other factors, such as the inhibition of inflammatory processes by regulatory mechanisms induced by helminths, to provide an explanation to the low lethality of COVID-19 in SSA. Interestingly, and probably in connection with it, the historical data relating to SARS-CoV and Middle East respiratory syndrome- $\mathrm{CoV}$ epidemics reveal that these viruses caused very limited health problems, if any, in Sub-Saharan countries (24). 
In a very recent paper, Bradbury et al., suggested that immune modulation by helminths could reduce the human resistance to SARS CoV-2 infection. Nevertheless, they called upon the research community to investigate whether helminth co-infection with COVID-19 could influence the pandemic spread through the helminth endemic regions of the world (25). Here, contrary to the opinion by Bradbury et al., we argue that helminth coinfection, in conjunction with at least part of the factors mentioned above, may be related to the low lethality of COVID-19 in SSA.

Furthermore, and looking ahead, we believe that helminth modulation on both type 1 and 2 immunity should be an

\section{REFERENCES}

1. World Health Organization. Coronavirus Press Conference. Geneva: WHO (2020).

2. Cucinotta D, Vanelli M. WHO declares COVID-19 a pandemic. Acta Biomed. (2020) 91:157-60. doi: 10.23750/abm.v91i1.9397

3. World Health Organization. Coronavirus disease 2019 (COVID-19): Weekly Epidemiological Update. Geneva: WHO. 27 p. (2020).

4. Wang Y, Wang Y, Chen Y, Qin Q. Unique epidemiological and clinical features of the emerging 2019 novel coronavirus pneumonia (COVID19) implicate special control measures. J Med Virol. (2020) 92:568-76. doi: $10.1002 / j m v .25748$

5. Wölfel R, Corman V, Guggemos W, Seilmaier M, Drosten C, Wendtner C. Virological assessment of hospitalized patients with COVID-2019. Nature. (2020) 581:465-9. doi: 10.1038/s41586-020-2196-x

6. Vabret N, Britton G, Gruber C, Hegde S, Kim J, Kuksin M, et al. Immunology of COVID-19: current state of the science. Immunity. (2020) 52:910-41. doi: 10.1016/j.immuni.2020.05.002

7. Qian Z, Travanty EA, Oko L. Innate immune response of human alveolar type II cells infected with severe acute respiratory syndrome-coronavirus. Am J Respir Cell Mol Biol. (2013) 48:742-8. doi: 10.1165/rcmb.2012-0339OC

8. Zhou Y, Fu B, Zheng X, Wang D, Zhao C. Pathogenic T cells and inflammatory monocytes incite inflammatory storm in severe COVID-19 patients. Natl Sci Rev. (2020) 7:nwaa041. doi: 10.1093/nsr/nwaa041

9. Xu Z, Shi L, Wang Y, Zhang J, Huang L, Zhang C, et al. Pathological findings of COVID-19 associated with acute respiratory distress syndrome. Lancet Resp Med. (2020) 8:420-2. doi: 10.1016/S2213-2600(20)30076-X

10. Napoli P, Nioi M. Global spread of Coronavirus Disease 2019 and Malaria: an epidemiological paradox in the early stage of a pandemic. J Clin Med. (2020) 9:1138. doi: 10.3390/jcm9041138

11. Ahmed S, Quadeer A, McKay M. Preliminary identification of potential vaccine targets for the COVID-19 Coronavirus (SARS-CoV2) based on SARS-CoV immunological studies. Viruses. (2020) 12:254. doi: 10.3390/v12030254

12. Gursel M, Gursel I. Is global BCG vaccination- induced trained immunity relevant to the progression of SARS- CoV-2 pandemic? Allergy. (2020) 75:1815-9. doi: 10.1016/j.mehy.2020.109707

13. Hotez PJ, Kamath A. Neglected tropical diseases in Sub-Saharan Africa: review of their prevalence, distribution, and disease burden. PLoS Negl Trop Dis. (2009) 3:e412. doi: 10.1371/journal.pntd.0000412

14. Saenz SA. Innate immune cell populations function as initiators and effectors in Th2 cytokine responses. Trends Immunol. (2010) 31:407-13. doi: 10.1016/j.it.2010.09.001

15. Taylor BC. TSLP regulates intestinal immunity and inflammation in mouse models of helminth infection and colitis. J Exp Med. (2009) 206:655-67. doi: $10.1084 /$ jem.20081499 important factor to consider during the design and evaluation of vaccines against SARS CoV-2 in those countries. The requirements of triggering type 1 responses for controlling viral replication and the development of type 2 immunopathology events observed during challenge experiments in animal models immunized with some coronavirus vaccine candidates support that reflection (26).

\section{AUTHOR CONTRIBUTIONS}

All authors listed have made a substantial, direct and intellectual contribution to the work, and approved it for publication.

16. Harris N, Loke P. Recent advances in type-2- cell- mediated immunity: insights from helminth infection. Immunity. (2017) 47:1024-36. doi: 10.1016/j.immuni.2017.11.015

17. Maizels RM. Regulation of Immunity and allergy by helminth parasites. Allergy. (2020) 75:524-34. doi: 10.1111/all.13944

18. Turner JD, Jackson JA, Faulkner H, et al. Intensity of intestinal infection with multiple worm species is related to regulatory cytokine output and immune hyporesponsiveness. J Infect Dis. (2008) 197:1204-12. doi: 10.1086/586717

19. Nacher M, Singhasivanon P, Traore B, Vannaphan S, Gay F, Chindanond D. Helminth infections are associated with protection from cerebral malaria and increased nitrogen derivatives concentrations in Thailand. Am J Trop Med Hyg. (2002) 66:304-9. doi: 10.4269/ajtmh.2002.66.304

20. Potian JA, Rafi W, Bhatt K, McBride A, Gause WC, Salgame P. Preexisting helminth infection induces inhibition of innate pulmonary anti-tuberculosis defense by engaging the IL-4 receptor pathway. J Exp Med. (2011) 208:186374. doi: $10.1084 /$ jem. 20091473

21. Furze R, Hussell T, Selkirk M. Amelioration of influenza-induced pathology in mice by coinfection with Trichinella spiralis. Infect Immun. (2006) 74:1924-32. doi: 10.1128/IAI.74.3.1924-1932.2006

22. Rolot M, Dougall AM, Chetty A, Javaux J, Chen T, Xiao X, et al. Helminthinduced IL-4 expands bystander memory CD8 + T cells for early control of viral infection. Nat Commun. (2018) 9:4516. doi: 10.1038/s41467-018-06978-5

23. McFarlane A, McSorley H, Davidson D, Fitch P, Errington C, Mackenzie K. Enteric helminth-induced type I interferon signaling protects against pulmonary virus infection through interaction with the microbiota. J Allergy Clin Immunol. (2017) 140:1068-78.e6. doi: 10.1016/j.jaci.2017.01.016

24. World Health Organization. Coronavirus Disease 2019 (COVID-19) Situation Report-70. Geneva: WHO. 16 p. (2020).

25. Bradbury R, Piedrafita D, Greenhill A, Mahanty S. Will helminth co-infection modulate COVID-19 severity in endemic regions? Nat Rev Immunol. (2020) 20:342. doi: 10.1038/s41577-020-0330-5

26. Enjuanes L, Zuñiga S, Castaño C, Gutierrez J, Canton L, Sola I. Molecular basis of coronavirus virulence and vaccine development. Adv Virus Res. (2016) 96:245-84. doi: 10.1016/bs.aivir.2016.08.003

Conflict of Interest: The authors declare that the research was conducted in the absence of any commercial or financial relationships that could be construed as a potential conflict of interest.

Copyright (C) 2020 Fonte, Acosta, Sarmiento, Ginori, García and Norazmi. This is an open-access article distributed under the terms of the Creative Commons Attribution License (CC BY). The use, distribution or reproduction in other forums is permitted, provided the original author(s) and the copyright owner(s) are credited and that the original publication in this journal is cited, in accordance with accepted academic practice. No use, distribution or reproduction is permitted which does not comply with these terms. 